Results Mean age was $81 \pm 4$ years, $60 \%$ were male and $88 \%$ received invasive treatment (percutaneous coronary intervention or coronary artery bypass grafting). At presentation, 39 $(16.8 \%)$ patients were robust, $123(53 \%)$ were pre-frail and $70(30.2 \%)$ were frail. Increasing frailty was associated with decreased physical QoL at both baseline and 1 year $(\mathrm{p}<0.001$ for both time points). Although all frailty groups saw an increase in mean PCS, this difference was only statistically significant in frail patients (robust: $42.4 \pm 11.4$ to 44.3 $\pm 12.4, \quad \mathrm{p}=0.372$; pre-frail: $38.4 \pm 11.4$ to $41.6 \pm 11.7$, $\mathrm{p}=0.117$; frail: $27.2 \pm 8.1$ to $32.9 \pm 12.7, \mathrm{p}=0.015)$. In addition, only frail patients who received invasive treatment saw this significant increase in PCS between baseline and 1 year, although numbers receiving medical therapy was low (Figure $1)$.

Conclusion Although frail older patients with NSTEACS have a poorer physical QoL overall, our data suggest frailty is associated with a similar or greater improvement from baseline QoL in those who receive invasive treatment.

\section{HIGH SERUM PARATHYROID HORMONE LEVELS ARE NOT ASSOCIATED WITH ENDOTHELIAL FUNCTION, VASCULAR STIFFNESS OR EARLY ADVERSE OUTCOMES AFTER INVASIVE MANAGEMENT OF NON-ST ELEVATION MYOCARDIAL INFARCTION IN HIGH-RISK OLDER PATIENTS}

\begin{abstract}
${ }^{1}$ Benjamin Beska, ${ }^{2}$ Dermot Neely, ${ }^{3}$ Harish Datta, ${ }^{1}$ Sophie Zhaotao Gu, ${ }^{1}$ Jonathan Batty, ${ }^{4}$ Hannah Sinclair, ${ }^{5}$ Guy MacGowan, ${ }^{6}$ Gary Ford, ${ }^{7}$ Weiliang Qiu, ${ }^{1}$ Vijay Kunadian*. ${ }^{1}$ Institute of Cellular Medicine, Faculty of Medical Sciences, Newcastle University; ${ }^{2}$ Department of Biochemistry, Newcastle upon Tyne Hospitals NHS Foundation Trust; ${ }^{3}$ Institute of Cellular Medicine, Newcastle University, and Department of Biochemistry, Newcastle upon Tyne Hospitals NHS Foundation Trust; ${ }^{4}$ Institute of Cellular Medicine, Newcastle University, and Cardiothoracic Centre, Freeman Hospital; ${ }^{5}$ Cardiothoracic Centre, Freeman Hospital, Newcastle upon Tyne Hospitals NHS Foundation Trust; ${ }^{6}$ Divison of Medical Sciences, Oxford University; ${ }^{7}$ Channing Division of Network Medicine, Department of Medicine, Brigham and Women's Hospital/Harvard Medical School
\end{abstract}

\subsection{6/heartjnl-2017-311726.54}

Introduction High serum parathyroid hormone (PTH) levels are associated with increased risk of cardiovascular events. Older patients have an increased risk of adverse events after non-ST elevation acute coronary syndrome (NSTEACS) and PTH may be a useful biomarker in these patients. The link between PTH, endothelial function, vascular stiffness and early outcomes after NSTEACS was evaluated.

Methods Older patients (65 years old) referred for invasive management of NSTEACS were recruited into the study $(n=204)$. Serum PTH was measured by electrochemiluminescent immunoassay and split into tertiles for analysis. Vascular stiffness was evaluated with carotid-femoral pulse wave velocity (PWV). Endothelial function was assessed by peripheral arterial tonometry, reported as natural log reactive hyperaemia index. Major Adverse Cardiovascular Events (MACE) were defined as 30 day composite of all-cause mortality, acute coronary syndrome, unplanned repeat revascularisation, significant bleeding, stroke or transient ischaemic attack. Multiple linear and logistic regressions were performed to control for age, sex, hypertension, diabetes, glomerular filtration rate and smoking status.

Results Mean age was $80.7 \pm 4.0$ years $(64.7 \%$ male). Median PTH was $5.9 \mathrm{pmol} / \mathrm{L}$ [IQR $4.3-7.8 \mathrm{pmol} / \mathrm{L}]$ and 81 patients (39.7\%) had levels above the normal range (1.1-6.4 pmol/L).
There were $83(40.7 \%)$ patients in the high $(6.4 \mathrm{pmol} / \mathrm{L}), 62$ $(30.4 \%)$ in the middle $(6.3-4.5 \mathrm{pmol} / \mathrm{L})$ and $59(28.9 \%)$ in the low tertile $(4.4 \mathrm{pmol} / \mathrm{L})$ of PTH. There was no difference in mean PWV (high 8.51 \pm 1.77 metres per second $(\mathrm{m} / \mathrm{s})$; middle $9.89 \pm 2.75 \mathrm{~m} / \mathrm{s}$; low $9.41 \pm 2.09 \mathrm{~m} / \mathrm{s} ; \mathrm{p}=0.646$ ) or mean natural $\log$ reactive hyperaemia index (high $0.64 \pm 0.34$; middle $0.61 \pm 0.23$; low $0.59 \pm 0.25 ; \mathrm{p}=0.684$ ) between PTH tertiles. There was no adjusted linear relationship between PTH and PWV $(p=0.09)$ or natural log reactive hyperaemia index $(p=0.919)$. MACE incidence did not vary between tertiles (high 2.4\%; middle 1.6\%; low 3.4\%; $\mathrm{p}=0.819$ ) and adjusting for covariates, PTH was not predictive of MACE $(p=0.308)$.

Conclusion In this high-risk older cohort, high serum PTH levels are not linked with endothelial dysfunction or vascular stiffness and do not predict early adverse events after invasive management of NSTEACS.

\section{HIGH SERUM PARATHYROID HORMONE LEVEL IS INDEPENDENTLY ASSOCIATED WITH CAROTID INTIMA- MEDIA THICKNESS IN OLDER PATIENTS UNDERGOING INVASIVE MANAGEMENT OF NON-ST ELEVATION MYOCARDIAL INFARCTION}

${ }^{1}$ Benjamin Beska, ${ }^{2}$ Dermot Neely, ${ }^{3}$ Harish Datta, 'Sophie Zhaotao Gu, 'Jonathan Batty, ${ }^{4}$ Hannah Sinclair, ${ }^{5}$ Gary Ford, ${ }^{6}$ Weiliang Qiu, ${ }^{1}$ Vijay Kunadian*. ${ }^{1}$ Institute of Cellular Medicine, Faculty of Medical Sciences, Newcastle University; ${ }^{2}$ Department of Biochemistry, Newcastle upon Tyne Hospitals NHS Foundation Trust; ${ }^{3}$ Institute of Cellular Medicine, Newcastle University, and Department of Biochemistry, Newcastle upon Tyne Hospitals NHS Foundation Trust; ${ }^{4}$ Institute of Cellular Medicine, Newcastle University, and Cardiothoracic Centre, Freeman Hospital; ${ }^{5}$ Divison of Medical Sciences, Oxford University; ${ }^{6}$ Channing Division of Network Medicine, Department of Medicine, Brigham and Women's Hospitall Harvard Medical School

\subsection{6/heartjnl-2017-311726.55}

Introduction Serum parathyroid hormone (PTH) levels, which are intimately linked to vitamin D status, are associated with an increased risk of cardiovascular events and mortality and may directly influence atherogenesis. Elevated carotid intimamedia thickness (CIMT) is a non-invasive marker of subclinical atherosclerosis and is associated with cardiovascular disease, providing predictive power above traditional risk factors. The association between PTH levels and CIMT was evaluated in older patients undergoing invasive management of non ST-elevation acute coronary syndrome (NSTEACS).

Methods High-risk older patients ( $n=160$, aged 65 years) attending a tertiary centre for invasive management of NSTEACS had CIMT of the left and right posterior carotid artery measured using B-mode ultrasound (Vivid- ${ }^{\circledR}{ }^{\circledR}$ GE Healthcare). The largest CIMT measurement was used for analysis. Serum PTH was measured by electrochemiluminescent immunoassay. Statistical modelling was performed using multiple regression, controlled by the hierarchical addition of a priori selected potential confounders.

Results Mean age was $80.4 \pm 4.0$ years (64.7\% male). Median PTH level was $5.6 \mathrm{pmol} / \mathrm{L}$ [IQR 4.0-6.8 pmol/L]. A significant relationship existed between logarithmically transformed serum PTH and CIMT (regression coefficient $(\mathrm{B})=0.230$, standard error of $\mathrm{B}(\mathrm{SEB})=0.086$, standardised regression coefficient $\left(\hat{\mathrm{I}}^{2}\right)$ $=0.208, p=0.008$ ) (Figure 1). The association was unchanged after adjustment for age, sex, glomerular filtration rate, body mass index, smoking status, hypertension and hypercholesterolemia $(B=0.219, \mathrm{SEB}=0.94, \beta=0.199, \mathrm{p}=0.021)$. Addition of serum vitamin $\mathrm{D}$ resulted in $\mathrm{a}<10 \%$ change in the regression 